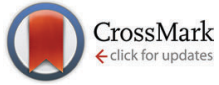

Cite this: J. Mater. Chem. C, 2015, 3, 6786

\title{
Bane to boon: tailored defect induced bright red luminescence from cuprous iodide nanophosphors for on-demand rare-earth-free energy-saving lighting applications $\uparrow$
}

\author{
Subhajit Saha, ${ }^{a}$ Swati Das, ${ }^{\text {b }}$ Dipayan Sen, ${ }^{\mathrm{b}}$ Uttam Kumar Ghorai, ${ }^{a}$ Nilesh Mazumder, ${ }^{b}$ \\ Bipin Kumar Guptac and Kalyan Kumar Chattopadhyay*ab
}

\begin{abstract}
The long standing controversy concerning the defect band in cuprous iodide (Cul) has been addressed in this paper from a technological point of view of its solid state lighting application. Recently, solid state lighting technology using nanophosphors has been proposed as the prime candidate in the energy saving lighting paradigm. Herein, we demonstrate a novel rare-earth free and non-toxic Cul nanophosphor, which has been synthesized via a facile solvothermal route. These nanophosphors are able to show ultrabright and stable red emission under near UV excitation. The spectral features of this easily derived nanophosphor are not less than any rare-earth or cadmium based conventional phosphor. Furthermore, it has been conclusively verified that the deep red emission is strongly related to the excess iodine induced optimized defect level engineering in the band structure. The concepts and results presented in this paper clearly establish that the Cul nanophosphor is a promising 'green' material for the state-ofthe-art rare-earth free lighting and display applications.
\end{abstract}

Received 13th March 2015, Accepted 20th May 2015

DOI: $10.1039 / c 5 t c 00719 d$

www.rsc.org/MaterialsC light with excellent color gamut. However, the relatively low efficiency of green LEDs and the different operating circuits for each individual RGB LED result in a poor price to performance ratio. An energetically and economically more efficient approach is to down-convert UV or blue light using a phosphor (or group of phosphors), such that they can collectively generate a white light. Unfortunately, these WLEDs suffer from some serious drawbacks such as a low color rendering index due to the poor efficiency of the red phosphor counterpart. ${ }^{5,6}$ To date, only a few red phosphors are available that can be excited using low energy. Therefore, recent consolidated efforts have been devoted to develop highly-luminescent red phosphors with superior spectral qualities that can be activated in the near UV region.

Doping rare-earth (RE) $\mathrm{Eu}^{3+}$ ions in a suitable host is traditionally the most popular protocol to achieve excellent red phosphors. ${ }^{7-9}$ The choice of this model originated from the notion that when $\mathrm{Eu}^{3+}$ occupies a non-centrosymmetric crystallographic site in the host lattice, it shows a strong characteristic red emission $(\lambda=\sim 615 \mathrm{~nm})$ because of the ${ }^{5} \mathrm{D}_{0} \rightarrow{ }^{7} \mathrm{~F}_{2} \mathrm{f}-\mathrm{f}$ transition. ${ }^{10}$ However, the production of these $\mathrm{RE}$ based phosphors not only requires high temperatures and pressures but also the use of RE ions as luminescence centers, exponentially increases the manufacturing cost of the device due to the scarcity of the elements. ${ }^{3,11,12}$ Further extensive and in depth search has unveiled highly luminescent red-light emitting CdSe or CdTe quantum dots as an alternative approach that 
might procure some advantages when compared with the traditional RE based red phosphors. Despite their conceived advantages, Cd based quantum dots have an inherently high level of toxicity even at relatively low concentrations, which significantly hinders their use in a practical scenario. ${ }^{13-15}$ Therefore, designing an environment-friendly nanophosphor at relatively low temperatures, which does not incorporate any rare-earth ion currently constitutes a 'Holy Grail' for WLED applications.

Herein, we report a novel RE-free and highly luminescent nanophosphor of cuprous iodide (CuI), whose luminescence properties are equally comparable with the conventional red phosphors. Due to the advent of high excitonic binding energy (62 $\mathrm{meV}$ ), the previous reports on $\mathrm{CuI}$ are predominantly focused on their room temperature free excitonic emissions. ${ }^{16,17}$ Most of these studies pay attention towards the inter band defect state elimination in CuI for their possible application in near UV excitonic LEDs. In this paper, for the very first time we are going to demonstrate that the precise modulation of the defect band inside the band structure of nanocrystalline CuI yields ultrabright visible luminescence in the deep red region. In the current study, a facile solvothermal route towards the synthesis of nanocrystalline $\mathrm{CuI}$ phosphors is presented. It is revealed that the forced incorporation of excess iodine in the CuI lattice results in a defect center mediated strong red emission, which has also been substantiated by the first principle calculations using atomistic density functional theory (DFT). The DFT results indicate that the intentional incorporation of interstitial iodine reduces the $\mathrm{Cu}-\mathrm{I}$ bond length, which facilitates the formation of inter-band states through mixing of the $\mathrm{p}$ and $\mathrm{d}$ states with the s orbital. This study puts light on the origin of the defect level emission in CuI, which is still under debate in the literature. The resulting ultra-bright red luminescence will promote $\mathrm{CuI}$ as a rare-earth free and environmentally safe nanophosphor for solid state lighting technology based on WLEDs.

\section{Results and discussion}

\subsection{Structural and compositional analysis}

We have carried out a detailed investigation of the structural and photophysical properties of the developed $\mathrm{CuI}$ nanophosphors at various stages of the solvothermal synthesis to study the effect of excess iodine on different physical parameters. Initially, white precipitates of CuI crystals were formed in the solution via the following reaction:

$$
2 \mathrm{CuCl}_{2}+4 \mathrm{KI}=2 \mathrm{CuI}+4 \mathrm{KCl}+\mathrm{I}_{2}
$$

Due to the presence of excess iodine, the solution was initially brown just before placing it in the autoclave. However, after 12 hour of reaction in a closed system, the solution became absolutely transparent (illustrated clearly in Fig. S1, ESI $\dagger$ ). This phenomenon indicates that the excess iodine dissolved in the reaction medium was incorporated into the precipitated $\mathrm{CuI}$ due to the application of high temperature and pressure. Confirmation of the phase purity of the obtained products was obtained by X-ray diffraction (XRD) analysis. The typical XRD pattern of the synthesized nanophosphors (shown in Fig. 1(a)) clearly reveals that all the diffraction peaks can be assigned to a cubic phase $\gamma$-CuI [JCPDS\#06-0246]. The wellassigned sharp diffraction peaks with no other unwanted signals confirm the phase purity of the products along with their highly crystalline nature.

A closer observation of the (111) XRD peak (shown in Fig. 1(b)) discloses the gradual lower angle shift of the peak position with an increase in the reaction time. Generally, such a shift in the XRD pattern is the result of unit cell expansion
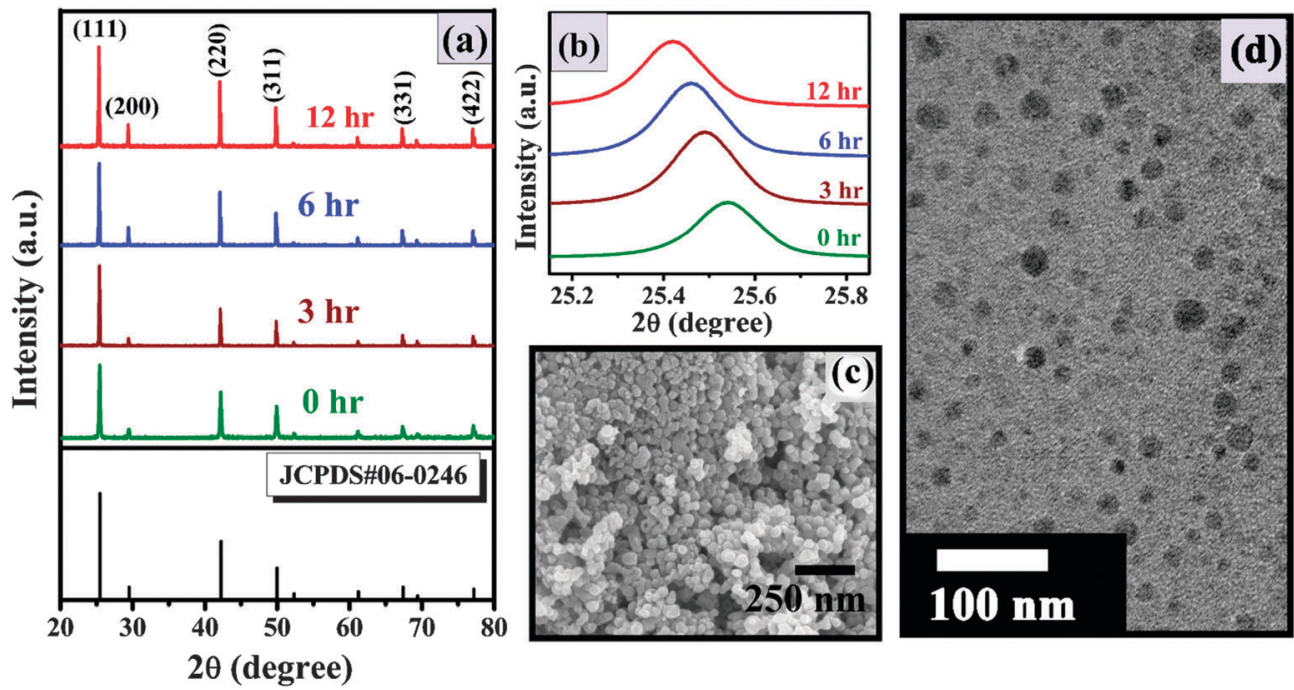

Fig. 1 (a) XRD pattern of Cul nanophosphors for different reaction times, representing the highly crystalline nature of the samples. (b) High resolution XRD pattern of the (111) peak, demonstrating a lower angle shift with the gradual increase in the reaction time. (c) The nanometer order size distribution of the Cul crystals. (d) TEM image, demonstrating the uniform dispersion of nanocrystalline Cul. 
caused by the substitution of larger atoms in the lattice. ${ }^{18,19}$ Similarly, in the current case, the incorporation of excess iodine in the CuI lattice is responsible for the increase in the unit cell volume as well as the consequent lower angle shift in the XRD pattern. The consistent variations in lattice parameter and unit cell volume (calculated from the XRD data) with the reaction time (shown in Fig. S2 of ESI $\dagger$ ) clearly support this logic. Since the expansion of the unit cell is always associated with a tensile strain, the occurrence of the proposed mechanism has been cross-checked by analyzing the induced lattice strain calculated from the Williamson-Hall plot, which is shown in Fig. S3 (in ESI $\dagger$ ). ${ }^{20}$ The trace of increasing lattice strain is clearly evident from continuous increase in the slope of the straight lines with reaction time. The presence of a uniform and regular narrow size distribution of these crystals within the nanometric region is prominent from the FESEM and TEM images, as shown in Fig. 1(c) and (d), respectively.

Further investigations of the exact chemical states of the constituent elements within the synthesized nanophosphors were carried out by detailed XPS analyses. All the core level spectra have been charge corrected by taking the peak of $\mathrm{C} 1 \mathrm{~s}$ at $284.6 \mathrm{eV}$, which appeared due to the presence of adventitious carbon on the surface of the samples during atmospheric exposure. The presence of $\mathrm{Cu}$ and I with no other impurity peaks in the typical survey scan (shown in Fig. S4(a), ESI $\dagger$ ) reflects the pure phase realization of CuI. The high resolution core level spectra for $\mathrm{Cu} 2 \mathrm{p}$ [shown in Fig. 2(a)] consists of two peaks at $952.1 \mathrm{eV}$ and $932.2 \mathrm{eV}$, which correspond to $\mathrm{Cu} 2 \mathrm{p}_{1 / 2}$ and $\mathrm{Cu} 2 \mathrm{p}_{3 / 2}$, respectively. The absence of any shake-up satellite peak in all the $\mathrm{Cu} 2 \mathrm{p}$ core level spectra strongly claims the +1 oxidation state of $\mathrm{Cu}$, which is the basic requirement for $\mathrm{CuI}$ formation. The peaks located at $619.7 \mathrm{eV}$ and $631.2 \mathrm{eV}$ [shown in Fig. 2(b)] are consistent with the I $3 d_{5 / 2}$ and I $3 d_{3 / 2}$ core level spectra of $\mathrm{I}^{-}$, respectively. Moreover, both the spin orbit split peaks for $\mathrm{Cu} 2 \mathrm{p}$ and I $3 \mathrm{~d}$ fit very well with only one component (shown in Fig. S4(b and c), ESI $\dagger$ ), which reflects the presence of only one valence state of the corresponding elements.

A careful inspection of the high resolution spectra of $\mathrm{Cu} 2 \mathrm{p}$ and I 3d clearly reveals that all the spectra are not exactly in the same binding energy position for all the synthesized nanophosphors, rather a little shift in their binding energy can be observed. The lower binding energy shift, along with the increased intensity of the I 3d peaks for the $12 \mathrm{~h}$ solvothermal treated samples, can be directly attributed to the presence of excess iodine in the corresponding nanophosphors. ${ }^{21,22}$ At the same time, a small higher binding energy shift can be noticed in $\mathrm{Cu} 2 \mathrm{p}$ peaks for the $12 \mathrm{~h}$ solvothermal treated samples. The incorporation of excess $\mathrm{I}^{-}$in the crystal reduces the electron density around $\mathrm{Cu}$, which in turn increases the binding energy value in the core level spectra. ${ }^{23}$ A detailed compositional analysis of the CuI nanophosphors synthesized at different solvothermal reaction times has also been carried out by studying their corresponding EDX (energy dispersive X-ray) spectra, which is shown in Fig. 2(c). EDX spectra clearly indicate a gradual increase in the I content within the nanocrystals with an increase in the reaction time and the ratio of $\mathrm{Cu}$ : I reaches a maximum of $1: 1.12$ for the samples synthesized via $12 \mathrm{~h}$ of solvothermal reaction. Therefore, the presence of excess iodine
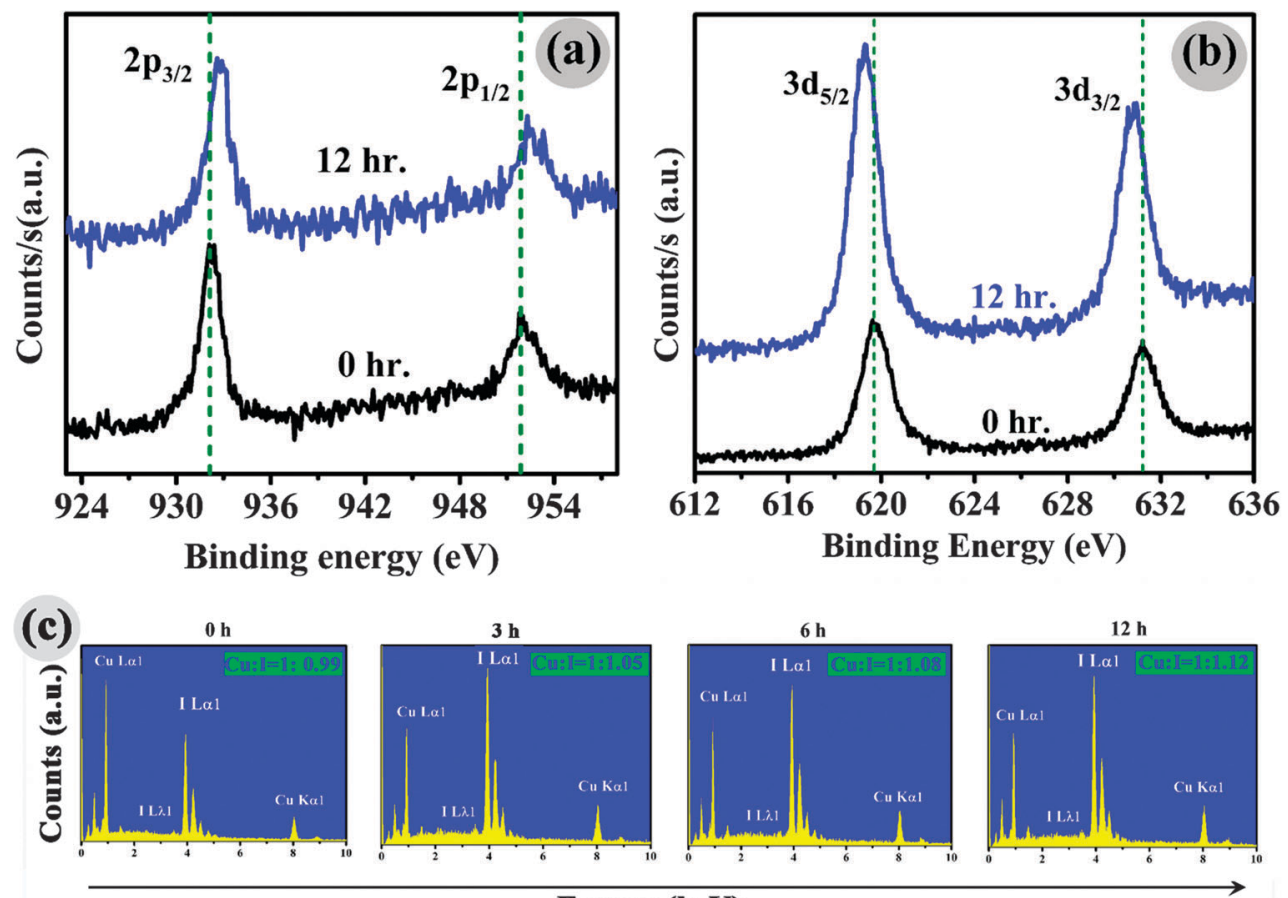

Energy (keV)

Fig. 2 Representation of the chemical shift in the high resolution XPS spectra of the Cu $2 p$ (a) and I 3d (b) core level of Cul nanophosphors. (c) EDX pattern of the Cul nanophosphors for different reaction times. 
in the synthesized nanophosphors was again confirmed by the EDX results.

\subsection{Luminescence study and DFT analysis}

To study the effect of iodine incorporation on the optical properties, room temperature photoluminescence (PL) spectra of the $\mathrm{CuI}$ nanophosphors have been obtained as a function of reaction time, which are presented in Fig. 3(a). The individual maxima in excitation/emission intensity were used to record the emission/excitation spectra. The excitation maximum is located at $397 \mathrm{~nm}$ (shown in Fig. S5 of ESI $\dagger$ ), which is one of the major excitation sources by the LED chips. The emission spectra primarily consist of two peaks; one weak peak at $\sim 421 \mathrm{~nm}$ and a broad peak centered at $688 \mathrm{~nm}$. The weaker peak at higher energy can be directly ascribed to the near band edge emission (NBE). ${ }^{16}$ Although the microscopic origin of the broad emission band remains unclear till date, ${ }^{24,25}$ the general consensus is that defect level mediated radiative recombination is responsible for the appearance of this red emission band. While the intensity of the defect level emission (DLE) increases gradually with reaction time, the NBE remains almost unaltered. The presence of strong DLE with a longer reaction time clearly demonstrates that these nanophosphors are very susceptible to various intrinsic defects and interstitials, which form new energy levels and screen the NBE emission. Moreover, these defects are generally distributed in the bodies of the nanophosphors. Experimental verification of this fact and the signature of the previously explained induced lattice strain in the nanophosphors can be realized straight through HRTEM analysis, as shown in Fig. 3(b). The HRTEM images of the nanophosphors obtained at two terminating points of the synthesis illustrate the difference between the lattice fringe patterns of the corresponding (111) planes. Initially, before placing into the autoclave the as-obtained samples were characterized by their highly crystalline nature with uniform and well-resolved lattice fringes with an interatomic spacing of $0.345 \mathrm{~nm}$. Consequently, the PL spectra of the samples show feeble DLE with a relatively stronger NBE emission. However, due to the application of a high pressure in the solvothermal reaction, the forced introduction of the excess iodine into the CuI crystals not only leaves the marks of defect induced lattice strain in the lattice fringes but also enhances the DLE of the corresponding samples. A signature of this defect induced lattice strain is distinctly detected by the high density of fault regions in the lattice fringes with slightly higher inter-atomic spacing $(\sim 0.351 \mathrm{~nm})$. This type of phenomenon is already supported by earlier reports with different nanophosphors, which show that the introduction of defects may distort the lattice fringes, leading to an enhancement of the DLE. ${ }^{26}$ Moreover, the calculated total density of states (TDOS) (shown in Fig. 3(c)) clearly demonstrate the appearance of impurity band near conduction band minima in the iodine incorporated samples. The recombination of electrons in the conduction band and holes in the valence band generates the NBE. However, when the electrons from these impurity levels

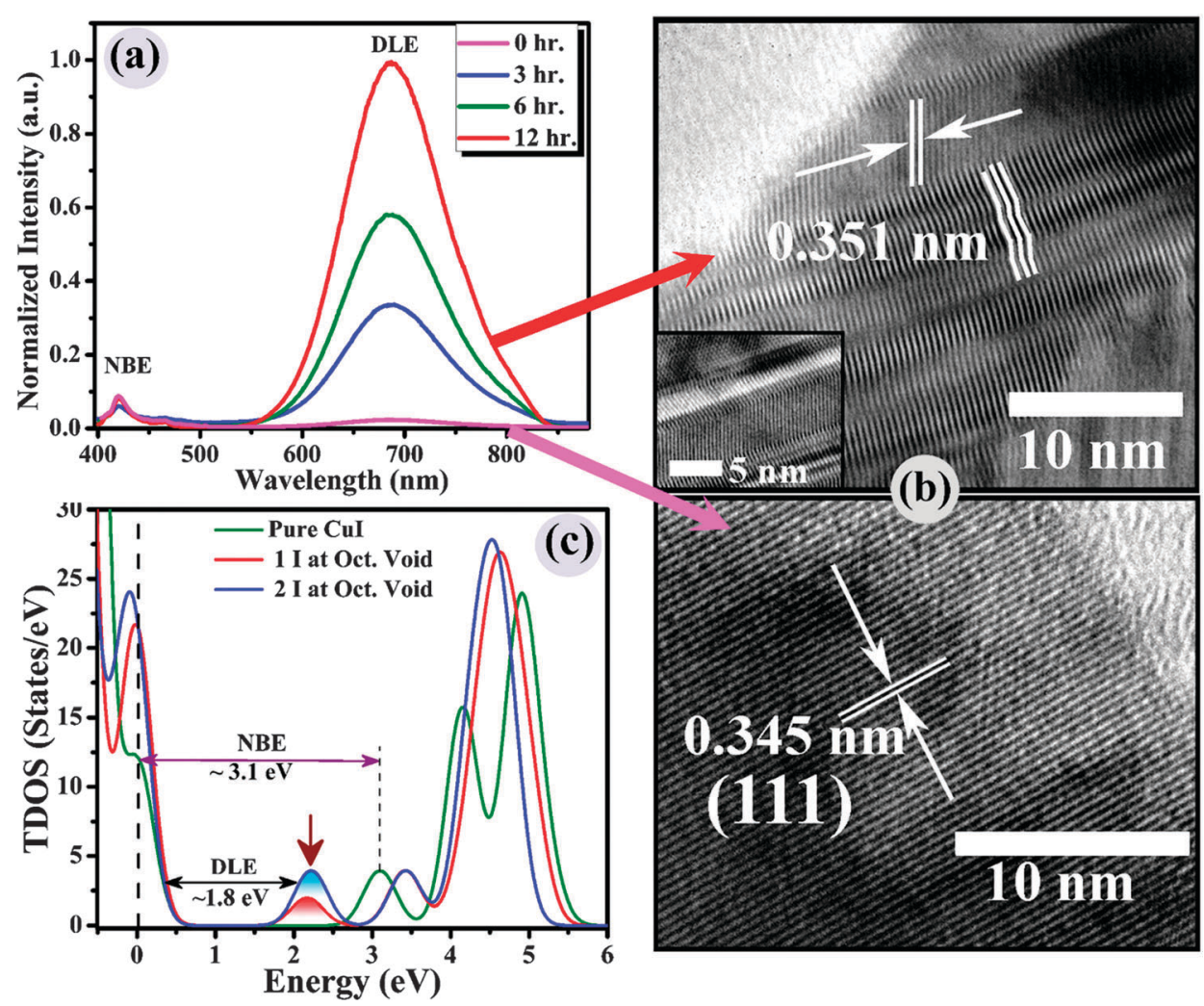

Fig. 3 (a) Room temperature PL spectra of the nanophosphors for different reaction times. (b) HRTEM images of the nanophosphors, showing the nature of lattice fringes. (c) Calculated total density of states (TDOS) for the pure and interstitial iodine incorporated Cul structures. 


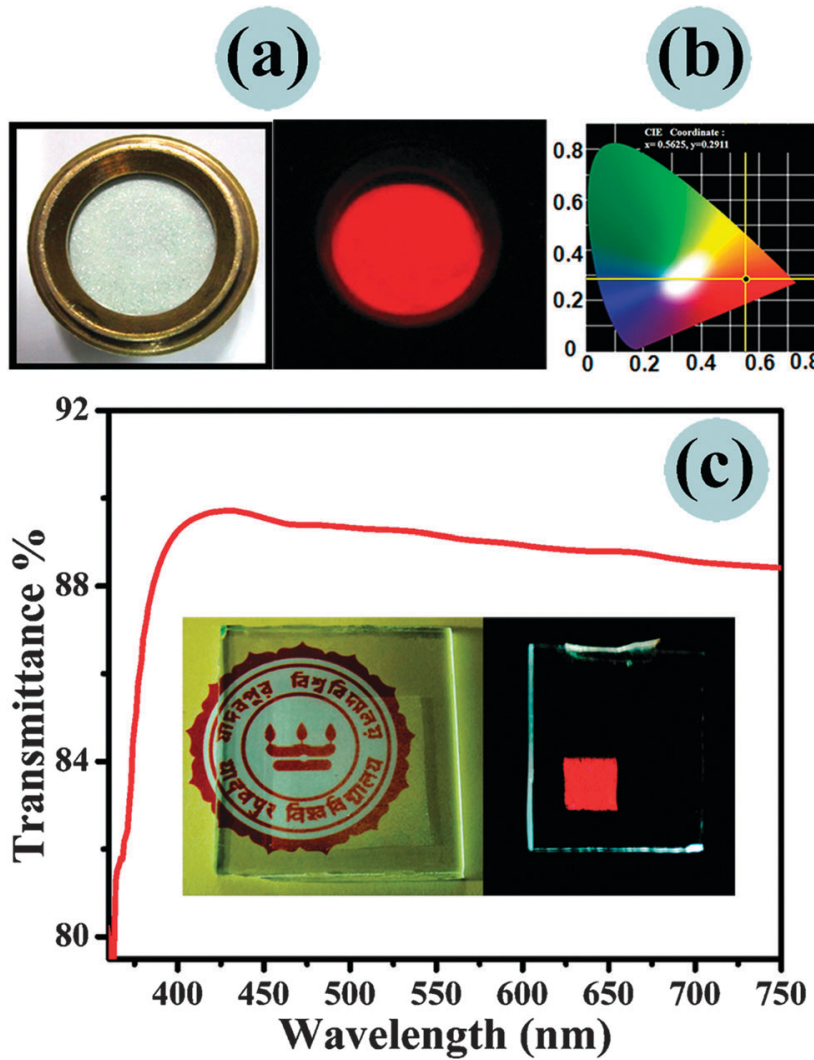

Fig. 4 (a) Optical images of the Cul nanophosphors irradiated under the visible and UV light, respectively. (b) Chromaticity diagram, representing the color coordinate of the corresponding red emission. (c) UV-Visible transmittance spectra of the Cul films. The inset represents the overall transparency of the films demonstrated by placing it over the logo of Jadavpur University and their bright emission under a 365 nm UV lamp.

recombine with the holes in the valence band, DLE takes place. Because the density of DLE is directly proportional to the density of the defect states, increasing incorporation of iodine increases these defect states as well as the DLE. The consistency of the PL spectra has been additionally verified by obtaining the cathodoluminescence (CL) spectra of the constituent samples, which are presented in Fig. S6 of the ESI. $\dagger$ Strong CL emission from the nanophosphors readily proves their potential to be used as field emission display phosphors.

The defect engineering-controlled high intensity ratio of DLE to NBE facilitates outstanding photoluminescence
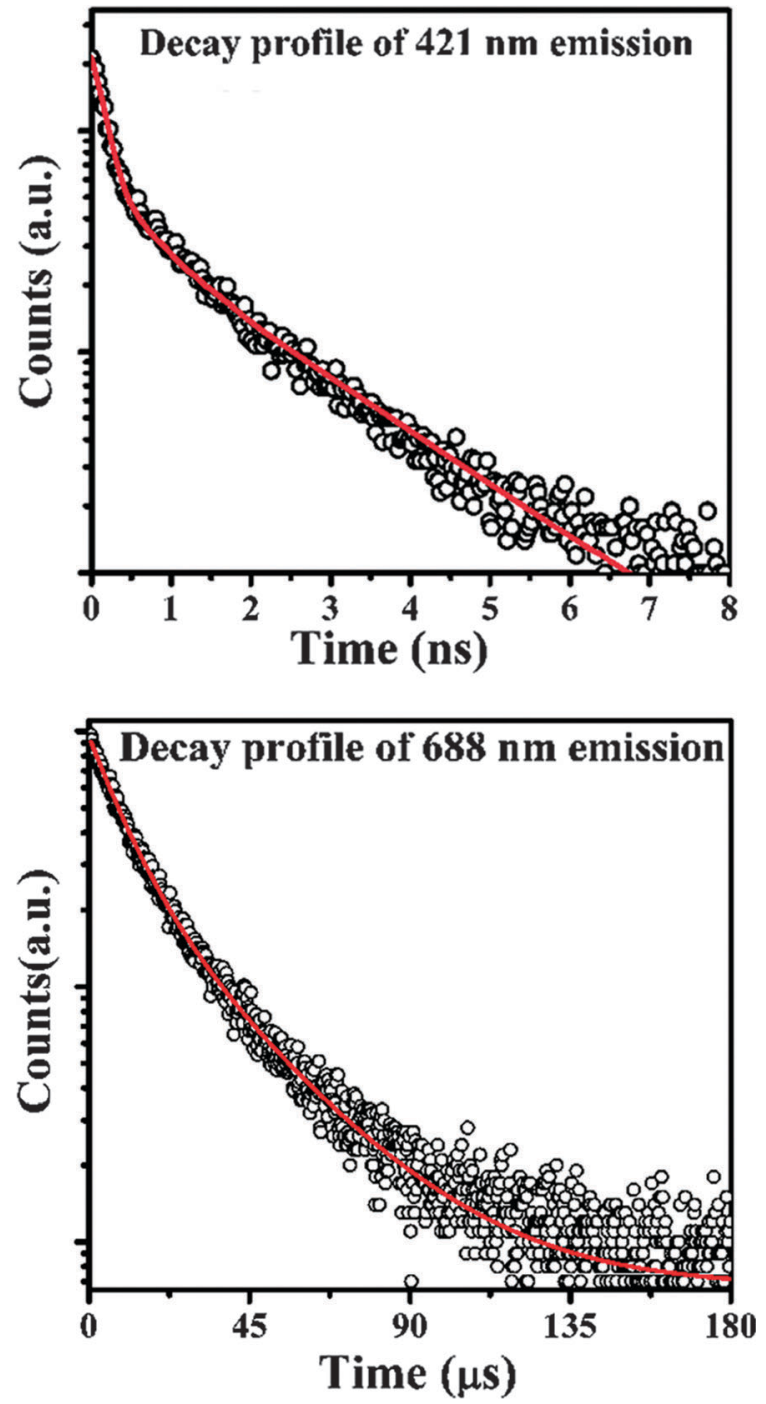

Fig. 5 Photoluminescence decay profiles of the nanophosphors for NBE and DLE.

properties from these CuI nanophosphors. The enhancement of DLE can be directly confirmed from the digital photograph of the nanophosphors under a $365 \mathrm{~nm}$ UV lamp (Fig. 4(a)). Upon UV exposure, the samples reveal an ultra-bright red emission whose color coordinate lies in the standard red region of the CIE chromaticity diagram (shown in Fig. 4(b)). Moreover, from the

Table 1 Comparison of the PL properties of the Cul nanophosphors with some already existing conventional red nanophosphors

\begin{tabular}{|c|c|c|c|c|c|}
\hline Nanophosphor type & $\begin{array}{l}\text { Excitation } \\
\text { wavelength }(\mathrm{nm})\end{array}$ & $\begin{array}{l}\text { Emission } \\
\text { wavelength }(\mathrm{nm})\end{array}$ & $\begin{array}{l}\text { PL quantum yield } \\
(\%)\end{array}$ & Remarks & Ref. \\
\hline $\mathrm{Y}_{2} \mathrm{O}_{3}: \mathrm{Eu}^{3+}$ nanophosphor & 253 & 611 & 10 (in solid) & $\begin{array}{l}\text { Costly, due to low excitation wavelength not } \\
\text { suitable for WLED }\end{array}$ & 32 \\
\hline $\mathrm{YVO}_{4}: \mathrm{Eu}^{3+}$ nanophosphor & 310 & 618 & 15 (in solution) & $\begin{array}{l}\text { Costly, due to low excitation wavelength not } \\
\text { suitable for WLED }\end{array}$ & 33 \\
\hline $\mathrm{YPO}_{4}: \mathrm{Eu}^{3+}$ nanophosphor & 350 & 613 & 0.31 (in solution) & Costly, poor quantum yield & 34 \\
\hline CdTe quantum dot & 385 & 652 & 15 (in solid) & Highly toxic & 29 \\
\hline CdS:Cu quantum dot & 460 & 663 & 18-30 (in solution) & Highly toxic & 27 \\
\hline Si quantum dot & 405 & 630 & 17 (in solution) & Low stability & 35 \\
\hline CuI nanophosphor & 397 & 688 & 21 (in solid) & Ecofriendly, non-toxic, stable & This work \\
\hline
\end{tabular}


application point of view, it is necessary to obtain phosphor thin films with enriched homogeneity and brightness. Interestingly, when the $\mathrm{CuI}$ nanocrystals are drop coated and blended on a glass substrate, under UV excitation these highly transparent films show a bright red emission, which is as strong as in their original powder form (shown in Fig. 4(c)). Since $\mathrm{Eu}^{3+}$ containing conventional red phosphors emit commonly below $630 \mathrm{~nm}$, it is very difficult to improve the color rendering index in the deep-red region using these phosphors. Therefore, the WLEDs fabricated using them also suffer from the problem of poor color rendering in the deep red region. ${ }^{27}$ However, the broad and intense red emission above $630 \mathrm{~nm}$, which is observed from the CuI nanophosphors, is expected to improve color rendering in the deep red region to a large extent. The PL quantum yield (QY) of these bright nanophosphors has been found to be $\sim 21 \%$ in the solid state. It should be notable that the enhanced surface states of the nanocrystals often quenches the PL QY in the solid form due to partial aggregation and energy transfer mediated non-radiative recombination. ${ }^{28,29}$ Therefore, it is very difficult to achieve a high quantum yield for the nanophosphor in the solid state. On the other hand, a smaller particle size is also needed to realize higher resolution for display technology. ${ }^{30,31}$ Keeping these points in mind, a careful survey regarding the PL properties of previously reported red nanophosphors is presented in Table 1 . From the table, it can be clearly perceived that the emission characteristics of this CuI nanophosphor are not only equally comparable with the red nanophosphors already reported in the literature but also it serves multifold advantages such as being ecofriendly, non-toxic and stable. The superior spectral characteristics along with uniform PL brightness throughout a large area promote this nanophosphor for near-UV activated WLEDs and display applications.

Further confirmation of the DLE has been accomplished using time-resolved photoluminescence (TRPL) measurements for both the emission wavelengths (shown in Fig. 5). The detailed fitting procedure for the corresponding decay curves along with different decay parameters are depicted in the ESI. $\dagger$ The average lifetime of NBE and DLE was found to be $1.37 \mathrm{~ns}$ and $2.82 \mu \mathrm{s}$, respectively. Because any emission originating from the defect states shows a longer lifetime than the intrinsic states, ${ }^{36}$ it is conclusively verified that the defect level created by the incorporation of excess iodine in the system generates a red luminescence band.

Density functional theory (DFT) calculations have also been carried out to analytically estimate the origin of the defect bands in the solvothermally grown CuI nanophosphors. Generally, at room temperature, CuI stabilizes in a zinc blende-like crystal structure. In such a structure, the $\mathrm{I}^{-}$ions form a cubic closed packed array in which half of the tetrahedral voids are occupied by the $\mathrm{Cu}^{+}$ions and all the octahedral voids remain empty. In a face centered cubic (FCC) lattice, the octahedral voids are located at the body center position and at the middle
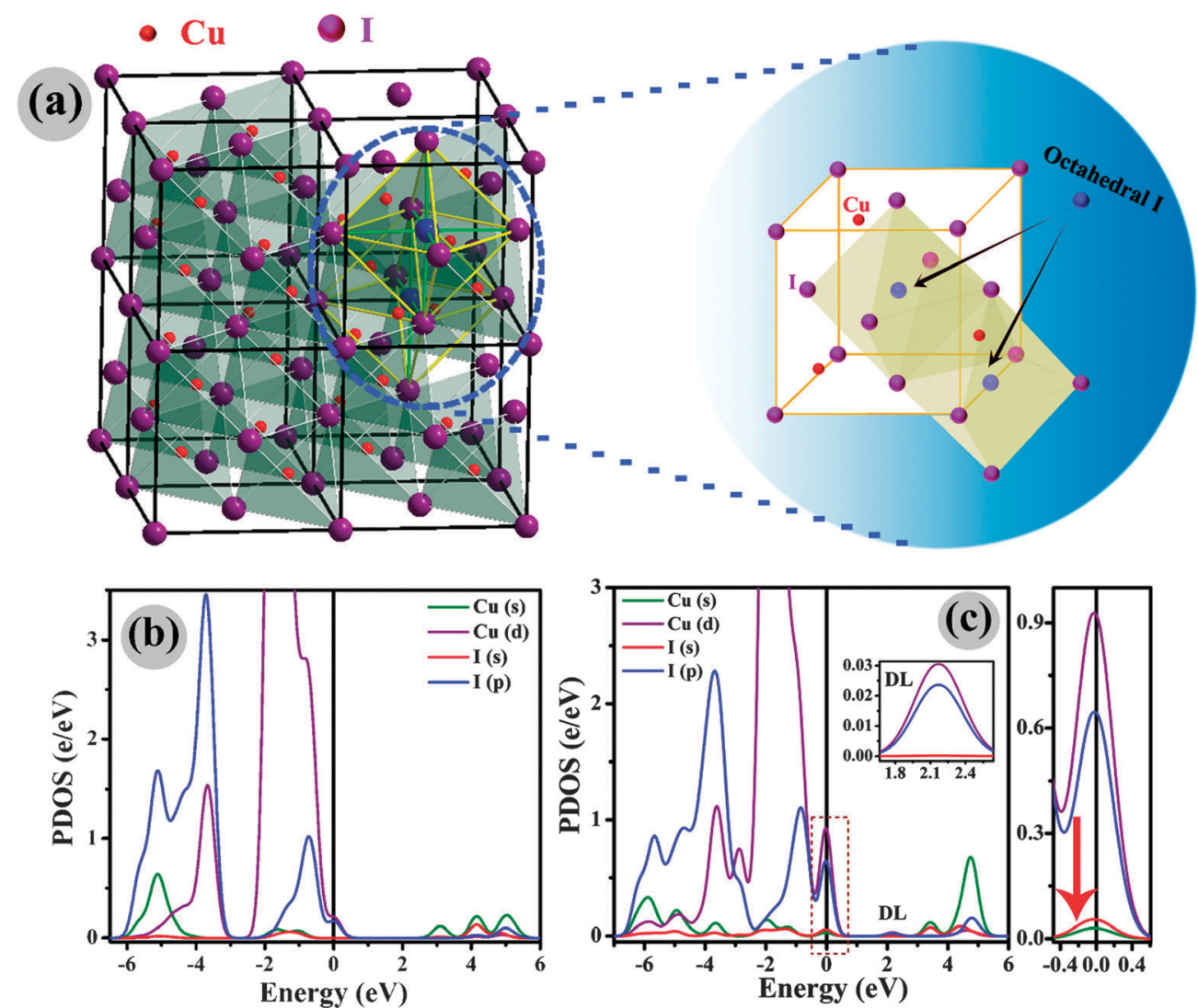

Fig. 6 (a) Polyhedral representation of the crytal structure of the Cul nanophophors. Magnified view of one unit cell that contains two excess iodine ions in the octahedral positions is also shown. (b) Partial density of states (PDOS) of the pure and (c) interstitial iodine incorporated Cul structures. 
of each edge. Therefore, to achieve our experimental conditions some extra iodine atoms would have to be incorporated in the crystal. Because the formation energy of an iodine interstitial at the tetrahedral site is considerably higher than an iodine interstitial at the octahedral site, ${ }^{37}$ we opted to introduce extra iodine ions in these octahedral sites (schematically shown in Fig. 6(a)). The corresponding complete supercell structures are supplied in Fig. S7 of the ESI $\dagger$ for reference. The addition of $\mathrm{I}^{-}$ in the octahedral sites alters the overall electronic properties of the crystal to a great extent, which is prominent from the TDOS patterns shown in Fig. 3(c). As expected, the TDOS pattern for pure CuI clearly demonstrates two distinct set of bands that are completely separated by a forbidden gap of $3.1 \mathrm{eV}$, which is in excellent agreement with the already reported band gap of $\gamma$ CuI. ${ }^{38,39}$ Moreover, the defect band that appears below $0.6 \mathrm{eV}$ from the conduction band minima in $\mathrm{I}^{-}$incorporated systems becomes increasingly stronger with the introduction of more iodine. To determine the exact orbital contributions that lead to the appearance of this defect band in $\mathrm{I}^{-}$incorporated systems, a precise probing of the TDOS curves was carried out by analyzing the partial density of states (PDOS) of the different orbitals, which are shown in Fig. 6(b) and (c). The PDOS patterns clearly confirm the well-known notion that the majority of the upper valence bands (around -2 to $0 \mathrm{eV}$ ) are dominated by
$\mathrm{Cu} 3 \mathrm{~d}$ with a noticeable contribution from I $5 \mathrm{p}$, whereas the lower edge of the conduction band consists of $\mathrm{Cu} 4 \mathrm{~s}$ along with an involvement of I 5s. As the spatial extent of the $\mathrm{Cu} 3 \mathrm{~d}$ levels is large and their energies are close to those of the I 5 p levels, these orbitals hybridize strongly to generate the valence band. The degree of $\mathrm{p}-\mathrm{d}$ admixture at the top of the valence band is 0.54 in CuI. ${ }^{40} \mathrm{~A}$ degree of $\mathrm{p}-\mathrm{d}$ hybridization is also prominent in both pure $\mathrm{CuI}$ and the iodine incorporated $\mathrm{CuI}$ system, as revealed in Fig. 6(b) and (c). A comparison of the PDOS patterns for both systems readily implies that the localized defect state, which appeared in the TDOS pattern of the I incorporated CuI structure, principally originates due to the major contribution from the $\mathrm{Cu} \mathrm{d}$ and I p orbitals (shown in the inset of Fig. 6(c)). However, the interesting fact is that, due to the introduction of iodine in the octahedral sites, the valence band becomes hybridized with an additional contribution from the $\mathrm{Cu} \mathrm{s}$ and I $\mathrm{s}$ orbitals, which is shown clearly in the right panel of Fig. 6(c). Such a mixing of $\mathrm{p}$ and $\mathrm{d}$ states with the s level is the result of the stronger overlap of the corresponding orbitals that occurred due to the reduction of the $\mathrm{Cu}-\mathrm{I}$ bond length. Initially, in pure $\mathrm{CuI}$, the $\mathrm{Cu}-\mathrm{I}$ bond length was $2.648 \AA$, whereas it changed to $2.553 \AA$ after the introduction of iodine in the octahedral sites. Moreover, this reduced bond length increases the density of states near the valence band edge due to the higher contribution from the
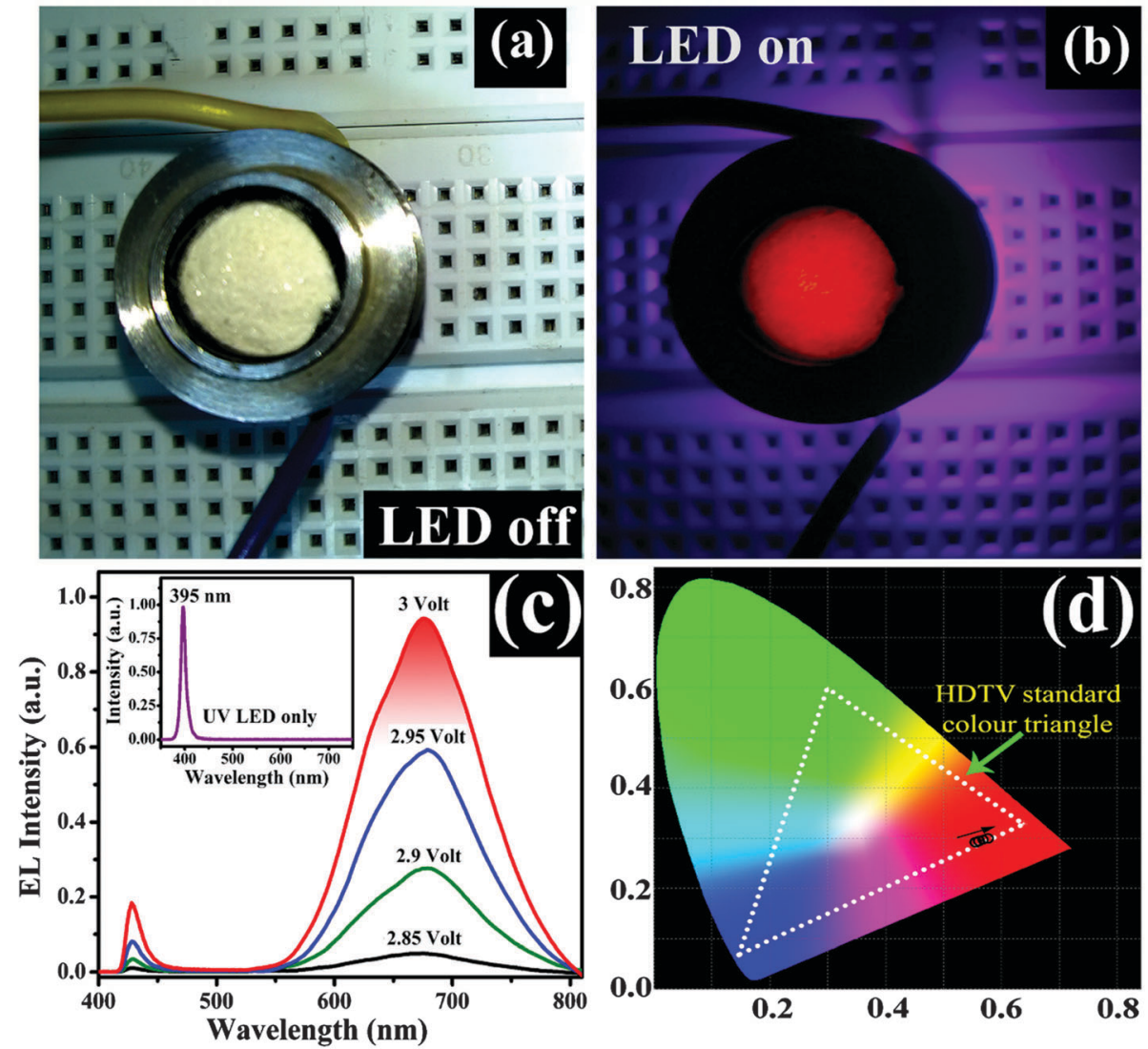

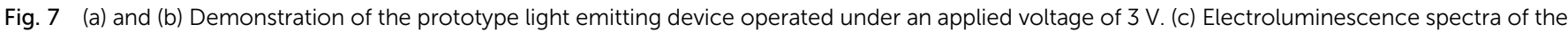

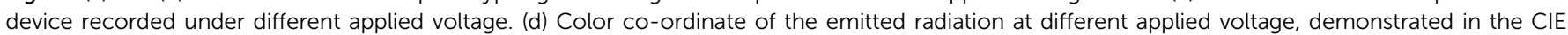
chromaticity diagram with respect to the HDTV standard color triangle. 
cationic $\mathrm{d}$ and anionic $\mathrm{p}$ levels. This mixing of the s level with $\mathrm{p}$ and $d$ states is responsible for the appearance of the localized defect states below the conduction band minima, as seen in the TDOS pattern. This type of phenomenon is already supported by earlier reports, which suggest that changes in the bond length can create new states within the band gap. ${ }^{41}$ Moreover, the existence of localized electronic levels near the conduction band can act as a trap for the excited electrons and is in accordance with the experimentally observed DLE in the room temperature PL spectra of the CuI nanophosphors.

After regular spectroscopic measurements, a prototype device was also constructed by adding the CuI nanophosphors over a commercial 395 nm UV LED, as represented in Fig. 7(a). Under an applied voltage of $\sim 3 \mathrm{~V}$, the device glows strongly and a bright red emission is observed (Fig. 7(b)). The typical electroluminescence (EL) spectra of the device obtained at different applied voltage are shown in Fig. 7(c). The position of the EL peaks is very close to their PL counterparts and with an increase in the applied voltage, the emission intensity increases gradually. The emission stability of the device was also checked and was found to fluctuate only $\sim 5 \%$ after more than 2 hours of operation (shown in Fig. S8(e) in ESI $\dagger$ ). Moreover, no spectral deterioration or appearance of extra peaks in the EL spectra was observed for the samples stored in the ambient conditions for more than 3 months. However, it is observed that the crystallinity and PL brightness of the nanophosphors decreases slightly after 3 months of storage (shown in Fig. S8(a-d), ESI $\dagger$ ). Due to such a long time of atmospheric exposure, the excess iodine present in CuI may easily react with atmospheric oxygen and undergo oxidization. A small peak corresponding to the (002) plane of $\mathrm{I}_{2} \mathrm{O}_{5}{ }^{42}$ also appeared in the XRD pattern (inset of Fig. S8(b), ESI $\dagger$ ) of the samples that was obtained after 3 months of storage. The oxidation is readily responsible for the reduction in the intensity observed in the XRD pattern and PL spectrum. The high color quality of the emitted light is demonstrated in the CIE chromaticity diagram, as shown in Fig. 7(d). With the variation of the applied voltage, the shift in the color coordinate was very small. Furthermore, the spectral locus of the emitted radiation lies on the high definition television (HDTV) standard color triangle, which strongly claims the color purity in terms of both hue and saturation.

\section{Conclusions}

In summary, highly luminescent and non-toxic CuI nanophosphors have been synthesized using a simple solvothermal route. By increasing the reaction time, the synthesized nanophosphors showed increased DLE with almost unaltered NBE. The red defect band is so strong that the samples show ultrabright red emission under a UV lamp both in the powder and thin film forms, which can be detected via the naked eye. By analyzing the HRTEM images, it was confirmed that structurally disordered nanophosphors show enhanced DLE. These disorders were attributed to the corresponding changes in the lattice created by the forceful introduction of iodine into the crystal structure. Further justification of such a direct relationship between structural disorder and PL properties was supported by the first principle calculations using density functional theory. DFT results clearly depict the evolution of a defect band within the forbidden bap in the interstitial iodine incorporated system. The theoretical calculations indicate that the introduction of interstitial iodine not only reduces the $\mathrm{Cu}-\mathrm{I}$ bond length but also facilitates the formation of inter-band states through mixing of $\mathrm{p}$ and $\mathrm{d}$ states with the s orbital. The obtained bright red emission with high quantum yield and good color rendering properties readily proves the potential of the CuI nanophosphors for using in WLEDs, display and general lighting applications. The present contribution takes a substantial step forward toward the burgeoning interest in the field of environment-friendly "rare-earth free solid state lighting" applications.

\section{Experimental section}

\subsection{Synthesis of CuI nanophosphors}

CuI nanophosphors have been synthesized using a solvothermal route. Analytical reagent grade $\mathrm{CuCl}_{2} \cdot 5 \mathrm{H}_{2} \mathrm{O}$ (Merck, 99.9\%), poly-ethylene-glycol (PEG), KI (Merck, 99.9\%), sodium dodecyl sulphate (Merck, 99.9\%) and $\mathrm{NaNO}_{3}$ (Merck, 99.9\%) were used as raw materials. The detailed synthesis procedure was as follows:

Initially, $0.05 \mathrm{M} \mathrm{CuCl}_{2} \cdot 5 \mathrm{H}_{2} \mathrm{O}$ was added to a $5 \mathrm{mM}$ PEG solution and stirred at $40{ }^{\circ} \mathrm{C}$ to obtain a clear solution. In a separate beaker, $0.1 \mathrm{M} \mathrm{KI}$ and $1.5 \mathrm{mM}$ SDS were mixed in a $5 \mathrm{mM}$ PEG solution. The mixture was stirred at $40{ }^{\circ} \mathrm{C}$ till the SDS in the PEG solution was complete dissolved and a transparent solution was obtained. This solution was then added dropwise to the abovementioned solution and stirred at $60{ }^{\circ} \mathrm{C}$ for $2 \mathrm{~h}$ until a viscous amaranth solution was obtained. The obtained solution was then added dropwise to a $0.05 \mathrm{M} \mathrm{NaNO}_{3}$ solution and stirred at $60{ }^{\circ} \mathrm{C}$ for $2 \mathrm{~h}$. Finally, tiny CuI crystals precipitated in the solution. This solution along with the precipitate was transferred to a $100 \mathrm{~mL}$ teflon-lined stainless steel autoclave and kept at $180{ }^{\circ} \mathrm{C}$ for several hours. After the reaction, the autoclave was allowed to cool naturally to room temperature and the precipitate was collected after washing several times with de-ionized water and ethanol. Finally, the nanophosphors were obtained by drying the obtained precipitate in an oven.

\subsection{Instrumentation}

The crystallinity and phase purity of the samples were examined by X-ray diffraction (XRD) using $\mathrm{Cu} \mathrm{K} \alpha$ radiation $(\lambda=$ $1.5406 \AA$ A ) (D8 Advanced, Bruker). The chemical state of the constituent elements were analyzed by X-ray photoelectron spectroscopy (XPS) using a monochromatic Al K $\alpha$ X-ray source $(h \nu=1486.6 \mathrm{eV})$ and a hemispherical analyzer (SPECS, HSA 3500). The morphology of the synthesized samples was examined using a field emission scanning electron microscope 
(FESEM Hitachi S-4800), while the crystalline structure of the individual samples was investigated using a high resolution transmission electron microscope (HRTEM JEOL JEM 2100). UV-Vis transmittance spectra were recorded on a Shimadzu UV3600 spectrophotometer. The steady state PL spectra were recorded on an Edinburgh, FLSP-980 spectrofluorometer, whereas TRPL measurements were carried out on IBH Fluorocube apparatus (Jobin Yvon-IBH-5000M) and Edinburgh, FLSP-980 luminescence spectrometer. The excitation and emission spectra were corrected for the spectral distribution of the Xe lamp intensity using a reference detector. Long pass optical filters have been used for the elimination of scattered excitation signal in the emission spectra. The photoluminescence quantum yield of the samples was measured using an integrating sphere whose inner face was coated with BENFLEC, as supplied by Edinburgh instruments. The cathodoluminescence (CL) spectra of the samples were recorded using Gatan Mono CL equipment attached to the FESEM using a beam accelerating voltage of $5 \mathrm{kV}$.

\subsection{Computational details}

Our first-principles calculations were performed by CASTEP code, which implements a supercell approach to density functional theory. The Perdew-Burke-Ernzerhof (PBE) functional within the generalized gradient approximation (GGA) was used to deal with exchange and correlation term. A Vanderbilt ultrasoft pseudopotential was used to represent the copper and iodine atoms, and plane waves up to an energy cut-off of $450 \mathrm{eV}$ was used in the calculation. Brillouin zone integrations were performed within the Monkhorst-Pack scheme using the $k$-point separation of $\sim 0.081 \times 0.081 \times 0.081 \AA^{-1}$. For geometrical optimization, the system was allowed to fully relax using the BFGS (Broyden-Fletcher-Goldfarb-Shanno) scheme until the total energy converged to less than $2 \times 10^{-5} \mathrm{eV}$ per atom, the maximum force was converged to lower than $0.05 \mathrm{eV}^{-1}$ and the maximum displacement was $0.002 \AA$. All the calculations were performed in a spin unrestricted manner.

To corroborate with the experimental results, two types of $\mathrm{CuI}$ structures were considered. For the pure CuI system, a $2 \times 2 \times 2$ supercell containing $32 \mathrm{Cu}$ atoms and $32 \mathrm{I}$ atoms was used, while to investigate the effect of interstitial iodine one and two iodine atoms were inserted in the octahedral sites. Prior to the calculation of the properties, all the systems were fully optimized in a spin unrestricted manner. A scissors operator of $2.174 \mathrm{eV}$ was used for all the electronic properties calculations to compensate for the inherent bandgap related problems associated with DFT.

\section{Acknowledgements}

The authors (SS, SD \& UKG) acknowledge the financial support from the Council of Scientific and Industrial Research (CSIR), the Government of India, for awarding Senior Research Fellowship during the execution of the work. One of us (DS) wishes to thank the W.B. State Govt. for providing fellowships during the execution of this work. We also wish to thank the Department of Science \& Technology (DST) and the University Grants Commission for 'University with Potential for Excellence scheme (UPE-II)', the Government of India, for financial help. Subhajit Saha, Swati Das and Dipayan Sen contributed equally to this work.

\section{References}

1 J. Z. Zhang, J. Phys. Chem. Lett., 2011, 2, 1351-1352.

2 C. C. Lin, Y. S. Zheng, H. Y. Chen, C. H. Ruan, G. W. Xiao and R. S. Liu, J. Electrochem. Soc., 2010, 157, H900.

3 C. C. Lin and R.-S. Liu, J. Phys. Chem. Lett., 2011, 2, 1268-1277.

4 E. F. Schubert and J. K. Kim, Science, 2005, 308, 1274-1278.

5 K.-S. Sohn, D. H. Park, S. H. Cho, J. S. Kwak and J. S. Kim, Chem. Mater., 2006, 18, 1768-1772.

6 E. Jang, S. Jun, H. Jang, J. Lim, B. Kim and Y. Kim, Adv. Mater., 2010, 22, 3076-3080.

7 C. Feldmann, T. Jüstel, C. R. Ronda and P. J. Schmidt, Adv. Funct. Mater., 2003, 13, 511-516.

8 C. Feldmann, Nanoscale, 2011, 3, 1947-1948.

9 H. A. Hoppe, Angew. Chem., 2009, 48, 3572-3582.

10 S. Saha, S. Das, U. K. Ghorai, N. Mazumder, B. K. Gupta and K. K. Chattopadhyay, Dalton Trans., 2013, 42, 12965.

11 T. Ogi, Y. Kaihatsu, F. Iskandar, W.-N. Wang and K. Okuyama, Adv. Mater., 2008, 20, 3235-3238.

12 X. Zhang, Y. Zhang, Y. Wang, S. Kalytchuk, S. V. Kershaw, Y. Wang, P. Wang, T. Zhang, Y. Zhao, H. Zhang, T. Cui, Y. Wang, J. Zhao, W. W. Yu and A. L. Rogach, ACS Nano, 2013, 7, 11234-11241.

13 M. Dasog, Z. Yang, S. Regli, T. M. Atkins, A. Faramus, M. P. Singh, E. Muthuswamy, S. M. Kauzlarich, R. D. Tilley and J. G. C. Veinot, ACS Nano, 2013, 7, 2676-2685.

14 M. J. Anc, N. L. Pickett, N. C. Gresty, J. A. Harris and K. C. Mishra, ECS J. Solid State Sci. Technol., 2012, 2, R3071-R3082.

15 C. Sun, Y. Zhang, Y. Wang, W. Liu, S. Kalytchuk, S. V. Kershaw, T. Zhang, X. Zhang, J. Zhao, W. W. Yu and A. L. Rogach, Appl. Phys. Lett., 2014, 104, 261106.

16 S. Das, S. Saha, D. Sen, U. K. Ghorai and K. K. Chattopadhyay, J. Mater. Chem. C, 2014, 2, 6592.

17 Z. Zheng, A. Liu, S. Wang, B. Huang, K. W. Wong, X. Zhang, S. K. Hark and W. M. Lau, J. Mater. Chem., 2008, 18, 852-854.

18 S. Zeng, G. Ren, C. Xu and Q. Yang, CrystEngComm, 2011, 13, 4276.

19 F. Ahmed, S. Kumar, N. Arshi, M. S. Anwar and B. Heun Koo, CrystEngComm, 2012, 14, 4016-4026.

20 G. K. Williamson and W. H. Hall, Acta Metall., 1953, 1, 22-31.

21 S. Ntais and A. Siokou, Surf. Sci., 2006, 600, 4216-4220.

22 S. Ntais, V. Dracopoulos and A. Siokou, J. Mol. Catal. A: Chem., 2004, 220, 199-205.

23 Y. Cong, J. Zhang, F. Chen and M. Anpo, J. Phys. Chem. C, 2007, 111, 6976-6982. 
24 A. Gruzintsev, JETP Lett., 2003, 78, 106-109.

25 (a) M. Gu, P. Gao, X. L. Liu, S. M. Huang, B. Liu, C. Ni, R.-K. Xu and J.-m. Ning, Mater. Res. Bull., 2010, 45, 636-639; (b) P. Gao, M. Gu, X. L. Liu, B. Liu and S.-M. Huang, Appl. Phys. Lett., 2009, 95, 221904; (c) A. N. Gruzintsev and V. N. Zagorodnev, Phys. Solid State, 2012, 54, 117-122; (d) G. P. Shevchenko, I. Y. Piskunovich, V. A. Zhuravkov and Y. V. Bokshits, Inorg. Mater., 2012, 48, 630-634; (e) M. Zi, J. Li, Z. Zhang, X. Wang, J. Han, X. Yang, Z. Qiu, H. Gong, Z. Ji and B. Cao, Phys. Status Solidi A, 2015, DOI: 10.1002/pssa.201532015.

26 H. Li, X. Wang, J. Xu, Q. Zhang, Y. Bando, D. Golberg, Y. Ma and T. Zhai, Adv. Mater., 2013, 25, 3017-3037.

27 X. Wang, X. Yan, W. Li and K. Sun, Adv. Mater., 2012, 24, 2742-2747.

28 T. Otto, M. Muller, P. Mundra, V. Lesnyak, H. V. Demir, N. Gaponik and A. Eychmuller, Nano Lett., 2012, 12, 5348-5354.

29 S. Kalytchuk, O. Zhovtiuk and A. L. Rogach, Appl. Phys. Lett., 2013, 103, 103105.

30 D. Geng, G. Li, M. Shang, C. Peng, Y. Zhang, Z. Cheng and J. Lin, Dalton Trans., 2012, 41, 3078.

31 G. Li, Z. Hou, C. Peng, W. Wang, Z. Cheng, C. Li, H. Lian and J. Lin, Adv. Funct. Mater., 2010, 20, 3446-3456.
32 R. Schmechel, M. Kennedy, H. von Seggern, H. Winkler, M. Kolbe, R. A. Fischer, L. Xaomao, A. Benker, M. Winterer and H. Hahn, J. Appl. Phys., 2001, 89, 1679-1686.

33 K. Riwotzki and M. Haase, J. Phys. Chem. B, 1998, 102, 10129-10135.

34 J. Chen, Q. Meng, P. S. May, M. T. Berry and C. Lin, J. Phys. Chem. C, 2013, 117, 5953-5962.

35 C.-C. Tu, J. H. Hoo, K. F. Böhringer, L. Y. Lin and G. Cao, Opt. Lett., 2012, 37, 4771-4773.

36 F. Liu, M. H. Jang, H. D. Ha, J. H. Kim, Y. H. Cho and T. S. Seo, Adv. Mater., 2013, 25, 3657-3662.

37 J. Wang, J. Li and S. S. Li, J. Appl. Phys., 2011, 110, 054907.

38 X. Hu, J. C. Yu, J. Gong and Q. Li, Cryst. Growth Des., 2007, 7, 262-267.

39 J. Wang, S.-S. Li, Y. Liu and J. Li, J. Phys. Chem. C, 2012, 116(2), 21039-21045.

40 A. Goldmann, J. Tejeda, N. Shevchik and M. Cardona, Phys. Rev. B: Condens. Matter Mater. Phys., 1974, 10, 4388-4402.

41 E. Longo, E. Orhan, F. M. Pontes, C. D. Pinheiro, E. R. Leite, J. A. Varela, P. S. Pizani, T. M. Boschi, F. Lanciotti, A. Beltrán and J. Andrés, Phys. Rev. B: Condens. Matter Mater. Phys., 2004, 69, 125115.

42 B. L. Zhu and X. Z. Zhao, Phys. Status Solidi A, 2011, 208(1), 91-96. 